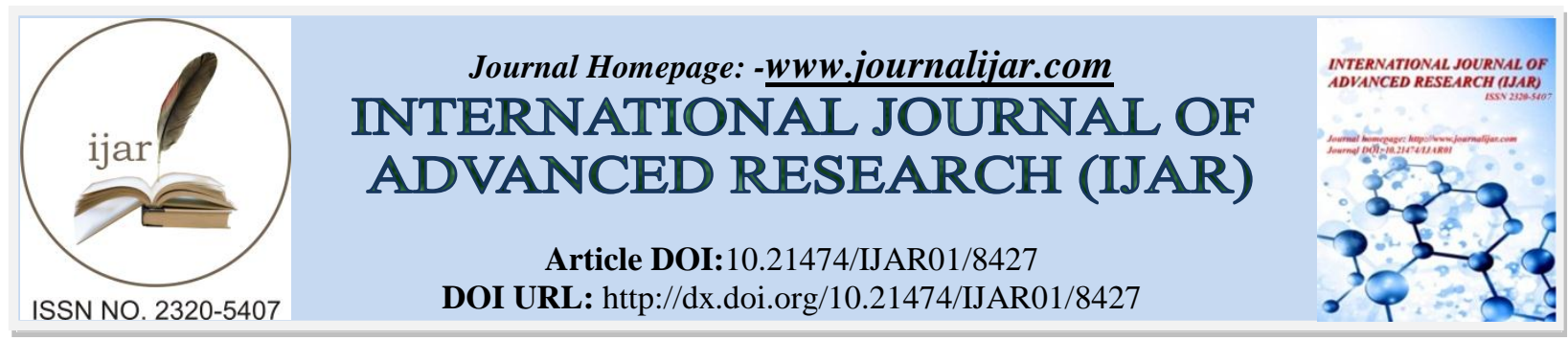

RESEARCH ARTICLE

\title{
EFFICACY OF EMERGENT ELECTROENCEPHALOGRAPHY (EMEEG) IN DETECTING NON- CONVULSIVE SEIZURES.
}

Sajeesh Parameswaran, Anilkumar T V, Vipin Radheyan, Lijo Johny C, Ajith M and A Marthanda Pillai Department of Neurosciences, Ananthapuri Hospitals and Research Institute, Trivandrum, Kerala, India.695024.

\section{Manuscript Info}

\section{Manuscript History}

Received: 16 November 2018

Final Accepted: 18 December 2018

Published: January 2019

Key words:-

Non-convulsive seizure, NCSE, EEG, EmEEG, Epilepsy.

\section{Abstract}

Introduction: Identification of non-convulsive seizures is important in neuro critical care practice. Emergent basis Electroencephalography (EmEEG) may helpful in detecting non-convulsive seizures and its medical management.

Objective: To assess the yield of EmEEG in detecting non-convulsive seizures.

Methods: Study was conducted in a tertiary level super specialty hospital. All patients entered in the emergent EEG register from June 2012 to December 2016 were included. 32 channels Digital EEG (Natus neurology, Canada) was used to perform EEG. Electrodes were placed according to 10-20 system. Clinical history, provisional diagnosis and other lab reports were analyzed.

Results: A total of 400 EEGs were analyzed. 40(10\%) patients showed periodic complexes, 33(8.3\%) patients showed non convulsive seizures, 20(5\%)patients showed non-convulsive status epilepticus, 13(3.3\%) patients showed complex partial seizures, 4 (1\%) patients showed statusepilepticus and 38(9.5\%) patients showed inter ictalepileptiform abnormalities. On the whole, out of 400 patients; 53 (13.25\%) showed non-convulsive seizures.

Conclusion:Emergent EEG has a major role in detecting nonconvulsive seizures and neuro-crtical care management.

Copy Right, IJAR, 2017,. All rights reserved.

\section{Introduction:-}

Routine electroencephalography (EEG) is considered as a planned procedure. Emergent EEG (EmEEG) is an EEG performed on emergent basis; upon request from an apparently emergency indication. EmEEG may help in diagnosis as well as in prognostication ${ }^{1}$. Most common reason for requesting EmEEG is to identify non-convulsive status epilepticus (NCSE) or electrographic seizures ${ }^{2,3}$. NCSE is a treatable medical condition, usually associated with subtle clinical signs or altered sensorium. It occurs in around $8 \%$ of comatose patients in intensive care units 4 and $37 \%$ of patients having unexplained altered consciousness ${ }^{5}$.

\section{Objective:-}

The aim of the present was to assess efficacy of emergent EEG in detecting non-convulsive seizures and to tabulate various EmEEG findings.

\section{Corresponding Author:-Sajeesh Parameswaran}

Address:-MSc, DNT, Electroencephalographer, Neuro Tech, Department of Neurosciences, 


\section{Methods:-}

We retrospectively analyzed all patients, who were entered in the EmEEG log book from June 2012 to December 2016. Only the first EmEEG record of each patient was included. Repeat studies of the same patient were excluded from the study. All EEG recordings were done on a 32 channel digital EEG acquisition system ( Xltek, Natus neurology, Canada). EEG scalp electrodes were placed according to internationally accepted 10-20 system. Standard activation procedures were employed whenever possible. Response to pain and other physiological stimuli were done in patients with altered sensorium. Minimum EmEEG recording period was 30 minutes. Time of EmEEG request, recording and reporting recorded. Clinical history, provisional diagnosis, imaging findings and other lab reports were tabulated. EEG criteria for NCSE followed according to the international guidelines ${ }^{6,7,8,9}$. Those patients with electrographic seizures and did not fulfill the criteria of NCSE were labeled as NCS.

Various EmEEG findings were tabulated and specific EmEEG findings which are helpful in management were noted. Quantitative EEG analysis (amplitude integrated EEG) was done in prolonged EmEEGs. Institute ethical committee was approved the study.

\section{Results:-}

A total of 400 EmEEGs were analyzed; which represent around 6\% of the total EEGs done in our lab. Age group was 1 month to 87 years; Male=240 Female=160. Major indication of EEG was either altered sensorium or coma. Out of 400 EmEEGs; 108 patients showed epileptiform abnormalities. 33(8.3\%) patients showed non convulsive seizures, 20(5\%) patients showed non-convulsive status epilepticus, $13(3.3 \%)$ patients showed complex partial seizures, $4(1 \%)$ patients showed status epilepticus and 38(9.5\%) patients showed inter ictalepileptiform abnormalities. On the whole, out of 400 patients; 53 (13.25\%) showed non-convulsive seizures.

Various other major EmEEG findings includes periodic complexes in 40 patients (10\%), electro cerebral inactivity in 45 patients (11.3\%) and different coma patterns in 16 patients (4\%). Hundred and sixty two patients labeled as non-specific dysfunction; which includes focal/diffuse slow waves, asymmetry and suppression. Twenty nine patient's (7.3\%) EmEEG found to be normal (Table 1).

\begin{tabular}{llll}
\hline No & Type & No of patients & Percentage \\
1 & Non-specific dysfunction(focal/diffuse) & 162 & $40.5 \%$ \\
2. & NCS/NCSE & 53 & $13.3 \%$ \\
3 & Seizure/Status epilepticus & 17 & $4.3 \%$ \\
4 & Inter ictalepileptiform abnormalities & 38 & $9.5 \%$ \\
5 & Periodic complexes & 40 & $10.0 \%$ \\
6 & Electro cerebral inactivity & 45 & $11.3 \%$ \\
7 & Alpha/theta/spindle coma & 16 & $4.0 \%$ \\
8 & Normal & 29 & $7.3 \%$ \\
\hline
\end{tabular}

Table 1:-Various EmEEG findings ( NCS- non convulsive seizure, NCSE- non convulsive status epilepticus).

Patient who had non-convulsive seizures were again classified according to etiology Out of 53 patients; in 20 patients $(37.7 \%)$ drug default was the major cause of the non-convulsive seizures. Metabolic disturbances identified in 9 patients (17\%) and various infections in 8 patients (15.1\%). Non-convulsive seizures seen in 10 patients with stroke (Table 2).

\begin{tabular}{lll}
\hline Etiology & No. of patients & Percentage \\
Epilepsy & 20 & $37.7 \%$ \\
Stroke & 10 & $18.9 \%$ \\
Metabolic disturbances & 9 & $17 \%$ \\
Traumatic brain injury & 3 & $5.7 \%$ \\
Anoxic brain injury & 3 & $5.7 \%$ \\
Infections (Encephalitis, meningitis, brain abscess, & 8 & $15.1 \%$ \\
sepsis) & & \\
\hline
\end{tabular}

Table 2:-Etiology - non-convulsive seizures. 
We categorized to two groups according to the age of the patient; group 1 (age $\geq 50$ years) and group 2 ( age $<50$ years). In the younger group 1; drug default and in group 2; metabolic/structural abnormalities were the major cause of the non-convulsive seizure $(p<.003)$. Different electrographic seizure patterns were identified. Focal onset non-convulsive seizure with clear evolution in frequency/amplitude (figure 1). Non-convulsive status epilepticus typical EEG patterns in patients without known epileptic encephalopathy as follows ;epileptiform Discharges $>2.5 \mathrm{~Hz}$ (figure 2). Amplitude integrated EEG was found to be useful in prolonged EmEEG recordings; especially to find frequency and duration of NCS. In a single window; we can visualize the entire recording. However; statistical significance was not studied (figure 3).

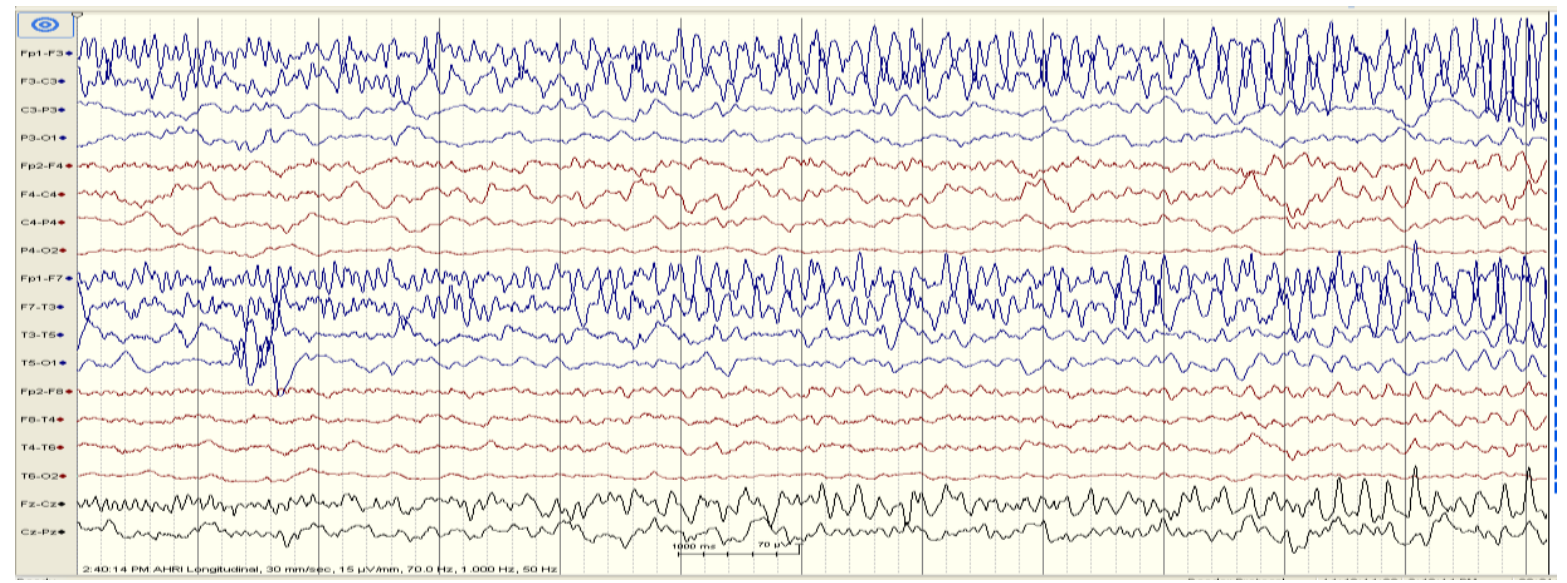

Figure 1:-focal onset non-convulsive seizure, with evolution in frequency/amplitude.

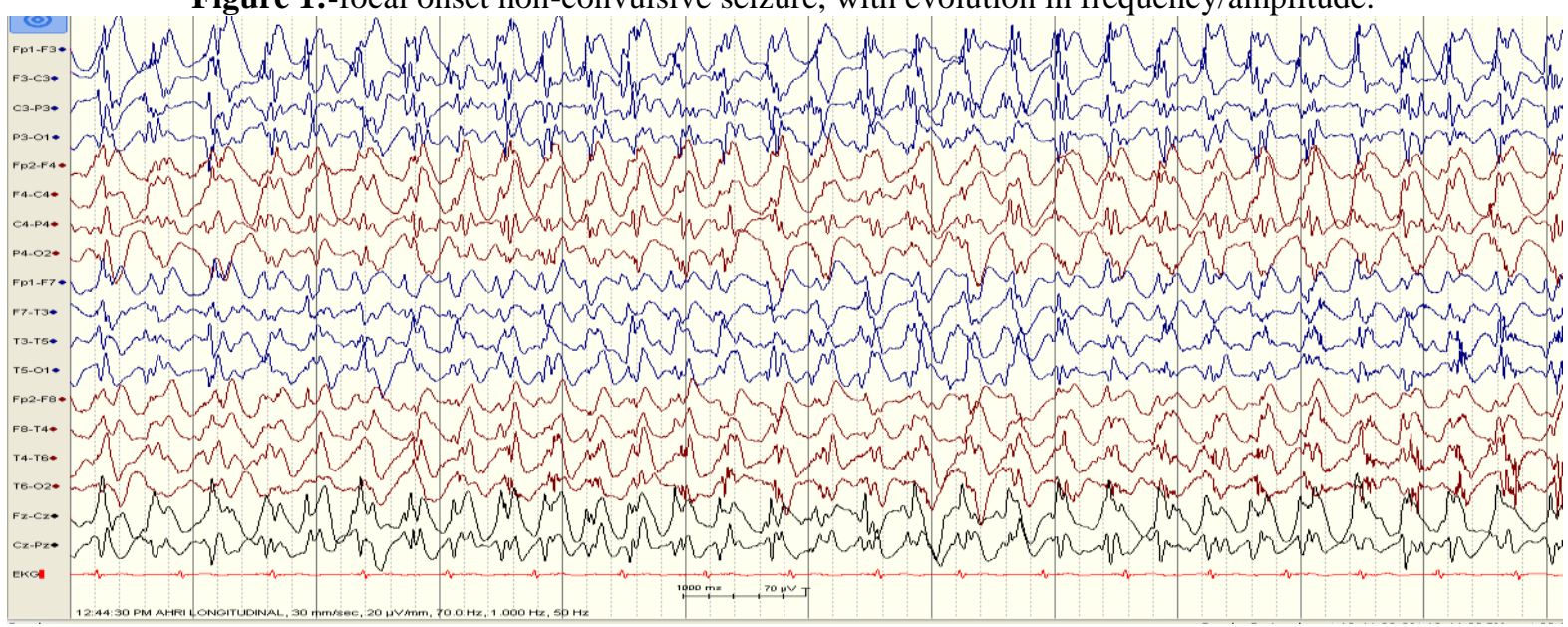

Figure 2:-Non convulsive status epilepticus, patient with altered sensorium ; spikes $>2.5 / \mathrm{sec}$ 


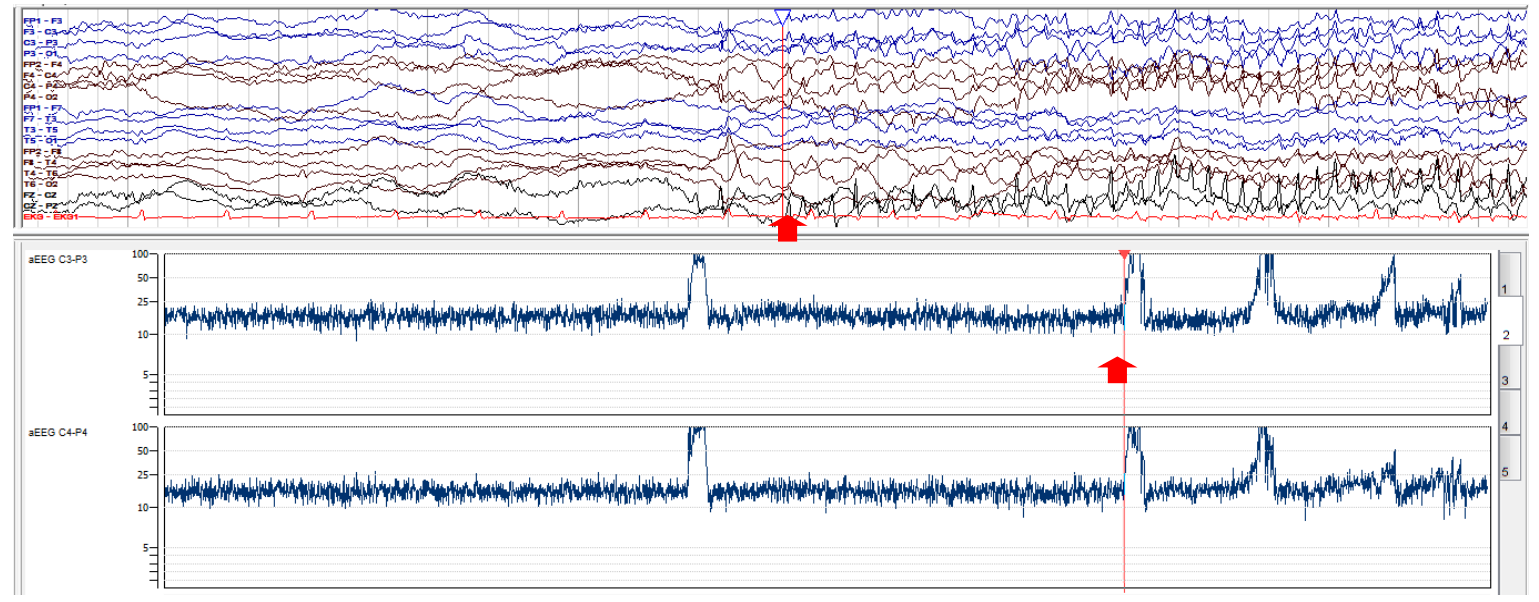

Figure 3:-Non convulsive seizures in amplitude integrated EEG (can easily identify frequency/duration of NCS in the prolonged recording).

\section{Discussion:-}

Nonconvulsive status epilepticus in which NCS are prolonged or repetitive, is variably defined as NCS lasting more than 30 minutes or recurrent over 30 minutes without return to normal consciousness; continuous or recurrent NCS lasting more than 5 minutes ${ }^{7}$. DeLorenzo et al. reported that NCSE continue in 14\% of patients following controlled generalized convulsive statusepilepticus ${ }^{8}$. NCSE is still under-diagnosed in the elderly and critically ill patients. NCSE should be terminated quickly to avoid patients from severe injuries, mainly if consciousness is impaired ${ }^{9}$. It is essential to distinguish NCSE early to avoid potential additional neuronal injury. NCSE is associated with considerable mortality and high morbidity ${ }^{10}$.

EmEEG contribute significantly to the treatment of patients with acute diseases, in particular those with changes in consciousness ${ }^{11}$. The presence of non-convulsive seizure in head trauma patients was reported ${ }^{12}$. EEG obtained in patients can frequently correlated with the etiology of diseases of the central nervous system. It can aid prognostication in the treatment of epileptic disorders, particularly if the etiology is known ${ }^{13}$.

Raw EEG data analysis is a time consuming procedure; whereas quantitative EEG analysis is useful in reducing the evaluation time by plotting seizures and trends ${ }^{14}$. In our study, we could clearly make out the importance of QEEG analysis in prolonged EmEEG monitoring. Graphical representation of the amplitude integrated EEG was much useful in identifying duration and frequency of non-convulsive seizure in the particular time interval.

Main aim of our study was to assess the incidence of non-convulsive seizures among patients who all were referred for EmEEG. Only few studies were documented in developing countries. The awareness and clinical correlation of EmEEG also very important. The 13.6\% diagnostic yield of EmEEG for non convulsive seizure in the present study would definitely highlight the importance of EmEEG and justify the need for extensive availability of EmEEG. However, patient selection for EmEEG is very important to increase the efficiency ${ }^{15,16,17}$. Etiology and age group analysis showed the importance of EmEEG in elder patients with metabolic disturbance; which can lead to NCSE and altered sensorium.

In our study we could identify various other EmEEG patterns like Intermittent Rhythmic Delta Activity (IRDA), Prolonged Bursts of Slow-wave Activity, Stimulus Induced rhythmic periodic of ictal discharges (SIRPIDs), Generalized Periodic Rhythmic Discharges, Lateralized Periodic Rhythmic Discharges, Triphasic Waves, Burst Suppression, Alpha and Theta Wave Coma, Sleep like Coma, Electro-cerbral inactivity etc. In the second phase of the study; we are planning to do a prospective analysis of patient's clinical condition, etiology and alteration in the diagnosis after the EmEEG findings. 


\section{Conclusion:-}

Emergent EEG is very useful diagnostic procedure in detecting non-convulsive seizures. Judicial use of EmEEG increases the efficacy. In elder population the most common reason for NCSE is either metabolic or structural etiology.

\section{References:-}

1. Khan SF, Ashalatha R, Thomas SV, Sarma PS. Emergent EEG is helpful in neurology critical care practice. Clinical neurophysiology. 2005 Oct 31;116(10):2454-9.

2. Praline J, Grujic J, Corcia P, Lucas B, Hommet C, Autret A, De Toffol B. Emergent EEG in clinical practice. Clinical Neurophysiology. 2007 Oct 31;118(10):2149-55.

3. Varelas PN, Spanaki MV, Hacein-Bey L, Hether T, Terranova B. Emergent EEG indications and diagnostic yield. Neurology. 2003 Sep 9;61(5):702-4.

4. Towne AR, Waterhouse EJ, Boggs JG, Garnett LK, Brown AJ, Smith J, DeLorenzo RJ. Prevalence of nonconvulsive status epilepticus in comatose patients. Neurology. 2000 Jan 25;54(2):340.

5. Privitera M, Hoffman M, Moore JL, Jester D. EEG detection of nontonic-clonic status epilepticus in patients with altered consciousness. Epilepsy research. 1994 Jun 30;18(2):155-66.

6. Trinka E, Cock H, Hesdorffer D, Rossetti AO, Scheffer IE, Shinnar S, Shorvon S, Lowenstein DH. A definition and classification of status epilepticus-Report of the ILAE Task Force on Classification of Status Epilepticus. Epilepsia. 2015 Oct 1;56(10):1515-23.

7. Trinka E, Leitinger M. Which EEG patterns in coma are nonconvulsive status epilepticus?. Epilepsy \& Behavior. 2015 Aug 31;49:203-22.

8. Leitinger M, Beniczky S, Rohracher A, Gardella E, Kalss G, Qerama E, Höfler J, Lindberg-Larsen AH, Kuchukhidze G, Dobesberger J, Langthaler PB. Salzburg consensus criteria for non-convulsive status epilepticus-approach to clinical application. Epilepsy \& Behavior. 2015 Aug 31;49:158-63.

9. Hirsch LJ, LaRoche SM, Gaspard N, Gerard E, Svoronos A, Herman ST, Mani R, Arif H, Jette N, Minazad Y, Kerrigan JF. American clinical neurophysiology society's standardized critical care EEG terminology: 2012 version. Journal of Clinical Neurophysiology. 2013 Feb 1;30(1):1-27.

10. Brophy GM, Bell R, Claassen J, Alldredge B, Bleck TP, Glauser T, LaRoche SM, Riviello JJ, Shutter L, Sperling MR, Treiman DM. Guidelines for the evaluation and management of status epilepticus. Neurocritical care. 2012 Aug $1 ; 17(1): 3-23$.

11. DeLorenzo RJ, Waterhouse EJ, Towne AR, Boggs JG, Ko D, DeLorenzo GA, Brown A, Garnett L. Persistent nonconvulsive status epilepticus after the control of convulsive status epilepticus. Epilepsia. 1998 Aug 1;39(8):83340 .

12. Meierkord H, Holtkamp M. Non-convulsive status epilepticus in adults: clinical forms and treatment. The Lancet Neurology. 2007 Apr 30;6(4):329-39.

13. Shneker BF, Fountain NB. Assessment of acute morbidity and mortality in nonconvulsive status epilepticus. Neurology. 2003 Oct 28;61(8):1066-73.

14. Ziai WC, Schlattman D, Llinas R, Venkatesha S, Truesdale M, Schevchenko A, Kaplan PW. Emergent EEG in the emergency department in patients with altered mental states. Clinical Neurophysiology. 2012 May 31;123(5):910-7.

15. Vespa PM, Nuwer MR, Nenov V, Ronne-Engstrom E, Hovda DA, Bergsneider M, Kelly DF, Martin NA, Becker DP. Increased incidence and impact of nonconvulsive and convulsive seizures after traumatic brain injury as detected by continuous electroencephalographic monitoring. Journal of neurosurgery. 1999 Nov;91(5):750-60.

16. Drislane FW. Presentation, evaluation, and treatment of nonconvulsive status epilepticus. Epilepsy \& Behavior. 2000 Oct 31;1(5):301-14.

17. Scheuer ML, Wilson SB. Data analysis for continuous EEG monitoring in the ICU: seeing the forest and the trees. Journal of clinical neurophysiology. 2004 Sep 1;21(5):353-78.

18. Quigg M, Shneker B, Domer P. Current practice in administration and clinical criteria of emergent EEG. Journal of Clinical Neurophysiology. 2001 Mar 1;18(2):162-5.

19. Claassen J, Taccone FS, Horn P, Holtkamp M, Stocchetti N, Oddo M. Recommendations on the use of EEG monitoring in critically ill patients: consensus statement from the neurointensive care section of the ESICM. Intensive care medicine. 2013 Aug 1;39(8):1337-51.

20. Abend NS, Dlugos DJ, Hahn CD, Hirsch LJ, Herman ST. Use of EEG monitoring and management of nonconvulsive seizures in critically ill patients: a survey of neurologists. Neurocritical care. 2010 Jun 1;12(3):382-9. 